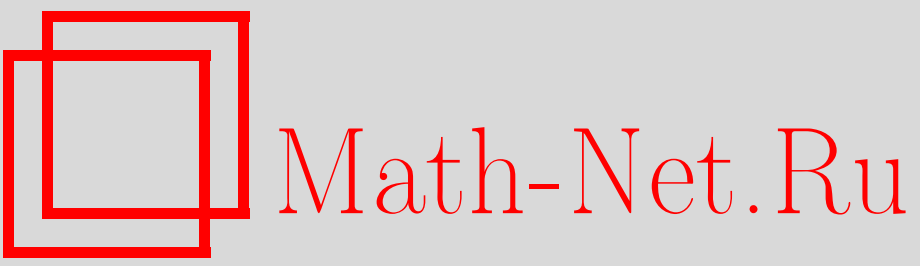

П. В. Ролдугин, А. В. Тарасов, О булевых функциях без верхних биюнктивных аналогов, Матем. вопр. криптогр., 2013, том 4, выпуск 1, 111-128

DOI: https://doi.org/10.4213/mvk76

Использование Общероссийского математического портала Math-Net.Ru подразумевает, что вы прочитали и согласны с пользовательским соглашением

http://www.mathnet.ru/rus/agreement

Параметры загрузки:

IP: 52.90.164.192

26 апреля 2023 г., 17:01:13 


\title{
О булевых функциях без верхних биюнктивных аналогов
}

\author{
П. В. Ролдугин, А. В. Тарасов \\ Московский государственный технический университет радиотехники, \\ электроники и автоматики, г. Москва \\ Получено 20.IV.2012
}

Изучаются условия на вес булевой функции, при которых она не имеет мажорирующих ее биюнктивных функций. Решаются комбинаторные перечислительные задачи, связанные с множествами таких булевых функций.

Ключевые слова: булевы функции, биюнктивные мажоранты, перечислительные задачи

On the Boolean functions without upper bijunctive analogues

\section{P. V. Roldugin, A. V. Tarasov}

Moscow State Technical University of Radio Engineering, Electronics and Automatics, Moscow

Abstract. Conditions on the weight of Boolean function of $n$ variables are found under which there are no bijunctive majorants of this function. Some combinatorial enumeration problems on the sets of such functions are solved.

Key words: Boolean functions, bijunctive majorants, enumeration problems

Citation: Mathematical Aspects of Cryptography, 2013, vol. 4, no. 1, pp. 111-128 (Russian). 


\section{Введение}

Будем использовать следующие обозначения и определения. Через $V_{n}$ обозначим множество двоичных векторов длины $n$. Для булевой функции $f$ от $n$ переменных обозначим через $E_{f}$ множество ее выполняющих векторов, то есть $E_{f}=\left\{\left(\alpha_{1}, \ldots, \alpha_{n}\right) \in V_{n}: f\left(\alpha_{1}, \ldots, \alpha_{n}\right)=1\right\}$. Через $\|\alpha\|$ обозначается вес вектора $\alpha \in V_{n}$; весом функции $f$ называется $\|f\|=\left|E_{f}\right|$. Имплицентой булевой функции $f$ называется (см. [1]) такая не равная константе булева функция $g$, что для любого значения набора переменных $\left(x_{1}, \ldots, x_{n}\right) \in V_{n}$ верно $f\left(x_{1}, \ldots, x_{n}\right) \cdot g\left(x_{1}, \ldots, x_{n}\right)=f\left(x_{1}, \ldots, x_{n}\right)$; иными словами, $E_{f} \subseteq E_{g}$.

Напомним, что биюнктивной функцией (см. [14]) называется булева функция $f$, представимая в виде $f=\stackrel{t}{i=1}_{s_{i 1}}^{t}\left(x_{s_{i 2}}^{\alpha_{i 1}} \vee x_{s_{i 2}}^{\alpha_{i 2}}\right), \quad \alpha_{i j} \in\{0,1\}, i \in\{1, \ldots, \mathrm{t}\}$, $j \in\{1,2\}$.

Иногда имплицента булевой функции $f$ называется верхним аналогом функции $f$ (см. [1]). В настоящей работе исследуются булевы функции, не имеющие в качестве имплицент биюнктивных функций, то есть не имеющие верхних биюнктивных аналогов.

\section{§ 1. Некоторые свойства булевых функций, не имеющих биюнктивных имплицент}

Функции, имеющие биюнктивные имплиценты, допускают простую характеризацию.

Лемма 1. Функиия $f$ имеет в качестве имплиценты биюнктивную функиию тогда и только тогда, когда она имеет имплиценту, зависящую не более чем от двух переменных.

Доказательство. Известно (см. [2]), что класс биюнктивных функций есть класс функций, представимых в виде произведения функций от двух или менее переменных. В частности, каждая функция от одной или двух переменных является биюнктивной. Отсюда сразу следует обратное утверждение леммы. Докажем прямое утверждение. Пусть функция $f$ имеет в качестве имплиценты биюнктивную функцию $g$. Тогда $g=\&_{i=1}^{s} g^{(i)}$, где каждая функция $g^{(i)}, i=1, \ldots, s$, зависит не более, чем от двух переменных, и для любых значений аргументов $f \cdot g=f$. Очевидно, что $g \cdot g^{(1)}=g$ 
и $f \cdot g^{(1)}=(f \cdot g) \cdot g^{(1)}=f \cdot\left(g \cdot g^{(1)}\right)=f \cdot g=f$. Следовательно, $g^{(1)}$ 一 имплицента функции $f$, зависящая не более чем от 2 переменных.

Зафиксируем значения $n, m, n \geq 1, m \geq 0$, и рассмотрим класс $D_{n, m}$ булевых функций от $n$ переменных, имеющих вес $m$ и не обладающих биюнктивными имплицентами. Учитывая, что вес функции от $n$ переменных не превосходит $2^{n}$, при $m>2^{n}$ верно равенство $D_{n, m}=\varnothing$.

Условию $m=0$ соответствует только функция, тождественно равная 0; эта функция, очевидно, обладает имплицентами от $n$ или менее переменных. Поэтому $D_{n, 0}=\varnothing$. Также очевидно, что каждая функция, не равная тождественно константе, является своей же имплицентой. Поэтому $D_{1, m}=\varnothing$ и $D_{2, m}=\varnothing$ по лемме 1 .

Рассмотрим множества $D_{n, m}$ при $1 \leq m \leq 3$ и произвольном $n \geq 1$. Пусть функция $f$ от $n$ переменных имеет вес $m$. Поскольку $m \leq 3$, найдется такая пара $(\mu, \eta) \in V_{2}$, что никакой вектор $\left(\mu, \eta, \alpha_{3}, \alpha_{4}, \ldots, \alpha_{n}\right)$ не лежит в $E_{f}$. Рассмотрим биюнктивную функцию $x_{1}^{\mu \oplus 1} \vee x_{2}^{\eta \oplus 1}$. Имеем

$$
\left(x_{1}^{\mu \oplus 1} \vee x_{2}^{\eta \oplus 1}\right) \cdot f\left(x_{1}, \ldots, x_{n}\right)= \begin{cases}0, & \text { если } x_{1}=\mu \text { и } x_{2}=\eta, \\ f\left(x_{1}, \ldots, x_{n}\right) & \text { в противном случае. }\end{cases}
$$

Но если $x_{1}=\mu$ и $x_{2}=\eta$, то $f\left(x_{1}, \ldots, x_{n}\right)=0$, значит, $\left(x_{1}^{\mu \oplus 1} \vee x_{2}^{\eta \oplus 1}\right) \cdot f\left(x_{1}, \ldots, x_{n}\right)=f\left(x_{1}, \ldots, x_{n}\right)$ при любых $\left(x_{1}, \ldots, x_{n}\right) \in V_{n}$. Следовательно, всякая функция веса $m, 1 \leq m \leq 3$, от $n$ переменных имеет биюнктивную имплиценту. Это означает, что $D_{n, m}=\varnothing$ при $1 \leq m \leq 3$.

Однако, как показывает следующее утверждение, для любого значения $m>3$ найдется такое значение $n$, что множество $D_{n, m}$ не пусто.

Утверждение 1. Пусть $m>3$. Тогда $D_{\left[\log _{2} m\right]+1, m} \neq \varnothing$.

Доказательство. Положим $n_{0}=\left[\log _{2} m\right]+1$. Поскольку $m=2^{\log _{2} m}<$ $<2^{\left[\log _{2} m\right]+1}=2^{n_{0}}$, то существуют булевы функции от $n_{0}$ переменных веса $m$. Докажем, что $D_{n_{0}, m} \neq \varnothing$, то есть покажем, что существует булева функция веca $m$ от $n_{0}$ переменных, не имеющая биюнктивных имплицент, или, что по лемме 1 то же самое, не имеющая имплицент от двух или одной переменной. 
Поскольку $m>3$, то $n_{0} \geq 3$. Рассмотрим функцию $f^{\prime}\left(x_{1}, \ldots, x_{n_{0}}\right)=$ $=x_{1} \oplus x_{2} \oplus x_{3}$. Очевидно, что $f^{\prime}$ не имеет имплицент от одной переменной: если $x_{i}^{\alpha} \cdot\left(x_{1} \oplus x_{2} \oplus x_{3}\right)=x_{1} \oplus x_{2} \oplus x_{3}, \alpha \in\{0,1\}, i \in\left\{1, \ldots, n_{0}\right\}$, то $x_{1} \oplus x_{2} \oplus x_{3}=0$ при $x_{i}=\bar{\alpha}$, что неверно, например, при $x_{1}=x_{2}=x_{3}=1$.

Предположим, что функция $g$ от двух переменных является имплицентой функции $f^{\prime}$. Для упрощения обозначений считаем, что $g$ зависит от переменных $x_{1}, x_{2}$. Имеем по определению $f^{\prime} \cdot g=f^{\prime}$. Значит,

$$
\begin{gathered}
f^{\prime}\left(x_{1}, \ldots, x_{n_{0}}\right) \cdot g\left(x_{1}, x_{2}\right)=\left(x_{1} \oplus x_{2} \oplus x_{3}\right) \cdot g\left(x_{1}, x_{2}\right)= \\
=\left(x_{1} \oplus x_{2}\right) \cdot g\left(x_{1}, x_{2}\right) \oplus x_{3} \cdot g\left(x_{1}, x_{2}\right)=f^{\prime}\left(x_{1}, \ldots, x_{n_{0}}\right)=x_{1} \oplus x_{2} \oplus x_{3} .
\end{gathered}
$$

Отсюда получаем, что

$$
\left(x_{1} \oplus x_{2}\right) \cdot \bar{g}\left(x_{1}, x_{2}\right)=x_{3} \cdot \bar{g}\left(x_{1}, x_{2}\right) .
$$

По определению имплиценты $g$ отлична от константы, следовательно, существуют такие $\alpha_{1}$ и $\alpha_{2}$, что $g\left(\alpha_{1}, \alpha_{2}\right)=0$. Выберем $\alpha_{3}=\alpha_{1} \oplus \alpha_{2} \oplus 1$. Подставляя вместо переменных $x_{1}, x_{2}, x_{3}$ значения $\alpha_{1}, \alpha_{2}, \alpha_{3}$, получаем из (1) равенство $\alpha_{1} \oplus \alpha_{2}=\alpha_{3}$, что противоречит выбору $\alpha_{3}$. Следовательно, у функции $f^{\prime}$ нет имплицент и от двух переменных и, следовательно, по лемме 1 , у функции $f^{\prime}$ нет биюнктивных имплицент.

У рассмотренной функции $f^{\prime}$ от $n_{0}=\left[\log _{2} m\right]+1$ переменных вес равен $2^{n_{0}-1}$. Докажем неравенство $0 \leq m-2^{n_{0}-1}<2^{n_{0}-1}$. Действительно, $n_{0}>\log _{2} m$, следовательно, $m<2^{n_{0}}$ или $m-2^{n_{0}-1}<2^{n_{0}-1}$. С другой стороны, $\log _{2} m \geq\left[\log _{2} m\right]=n_{0}-1$, поэтому $m \geq 2^{n_{0}-1}$ или $0 \leq m-2^{n_{0}-1}$.

Добавим к множеству $E_{f^{\prime}}$ еще $m-2^{n_{0}-1}$ новых различных $n_{0}$-мерных двоичных векторов, выбрав их произвольно (это возможно сделать именно ввиду неравенства $\left.0 \leq m-2^{n_{0}-1}<2^{n_{0}-1}\right)$. Полученное множество является множеством выполняющих векторов некоторой функции $f$ от $n_{0}$ переменных и веса $m$. Предположим, что функция $g$ является имплицентой функции $f$. Имеем по определению $E_{f} \subset E_{g}$; поскольку по построению $E_{f^{\prime}} \subset E_{f}$, то $E_{f^{\prime}} \subset E_{g}$, то есть $g-$ имплицента функции $f^{\prime}$. Но, как показано выше, функция $f^{\prime}$ не имеет имплицент от двух или менее переменных. Получаем, что и у функции $f$ нет таких имплицент. Таким образом, $D_{n_{0}, m} \neq \varnothing$.

Далее рассмотрим случай, когда $n$ достаточно велико. 
Утверждение 2. Пусть $m>0$. Тогда для любых значений $n, n>2^{m}$, $D_{n, m}=\varnothing$.

Доказательство. Пусть $m, n \in \mathrm{N}$. Докажем, что, если $n>2^{m}$, то у любой функции $f$ от $n$ переменных и веса $m$ найдется имплицента, зависящая не более чем от 2 переменных. Согласно лемме 1 тогда существует биюнктивная имплицента; это и означает, что при $n>2^{m}$ все множества $D_{n, m}$ пусты. Рассмотрим произвольную функцию $f\left(x_{1}, \ldots, x_{n}\right)$ веса $m$. Пусть $E_{f}=\left\{\left(\alpha_{1}^{(i)}, \ldots, \alpha_{n}^{(i)}\right): i=1, \ldots, m\right\}-$ множество ее выполняющих векторов. Рассмотрим для каждого $j=1, \ldots, n$ булевы векторы $\left(\alpha_{j}^{(1)}, \alpha_{j}^{(2)}, \ldots, \alpha_{j}^{(m)}\right)$. Это векторы размерности $m$, и их $n$ штук. Так как $n>2^{m}$, то среди указанных векторов найдутся два одинаковых вектора $\left(\alpha_{u}^{(1)}, \alpha_{u}^{(2)}, \ldots, \alpha_{u}^{(m)}\right)=$ $=\left(\alpha_{v}^{(1)}, \alpha_{v}^{(2)}, \ldots, \alpha_{v}^{(m)}\right), 1 \leq u<v \leq n$. Это означает, что если $f\left(x_{1}, \ldots, x_{n}\right)=1$, то $x_{u}=x_{v}$. Тогда функция $g=x_{u} \vee \bar{x}_{v}$ является имплицентой функции $f$. Действительно, если $f\left(x_{1}, \ldots, x_{n}\right)=1$, то $x_{u}=x_{v}$ и, следовательно, $g=x_{u} \vee \bar{x}_{v}=x_{u} \vee \bar{x}_{u}=1$. Значит, $E_{f} \subseteq E_{g}$, то есть $g-$ имплицента функции $f$.

Согласно утверждениям 1 и 2 для любого фиксированного $m>3$ в последовательности множеств $D_{3, m}, D_{4, m}, D_{5, m}, \ldots$ найдется непустое множество и, вместе с тем, начиная с некоторого номера, все множества пусты. Это обосновывает корректность следующего определения.

Определение. При фиксированном $m \geq 4$ положим величину $n_{\max }(m)$ равной наибольшему значению $n$, при котором $D_{n, m} \neq \varnothing$.

Дополнительно по определению положим $n_{\max }(1)=n_{\max }(2)=n_{\max }(3)=0$.

Определение величины $n_{\max }(m)$ можно переформулировать так: это наибольшее возможное количество переменных булевой функции веса $m$, не имеющей верхних биюнктивных аналогов.

Из доказанных утверждений получаем оценку для величины $n_{\max }(m)$.

Следствие 1. При $m>3$ верны неравенства $0<n_{\max }(m) \leq 2^{m}$.

Отметим свойство монотонности функции $n_{\max }(m)$, которое понадобится далее.

Утверждение 3. Если $m>0$, то $n_{\max }(m) \leq n_{\max }(m+1)$. 
Доказательство. Рассмотрим произвольное значение $n$, для которого $n \geq\left[\log _{2} m\right]+1$ и $D_{n, m} \neq \varnothing$ (по утверждению 1 хотя бы одно такое значение $n$ существует). Далее возьмем произвольную функцию $f \in D_{n, m}$. Имеем $\|f\|=m=2^{\log _{2} m}<2^{\left[\log _{2} m\right]+1} \leq 2^{n}$. Так как $m<2^{n}$, то существует вектор $\left(\alpha_{1}, \ldots, \alpha_{n}\right)$, который не лежит в множестве $E_{f}$. Добавим в $E_{f}$ вектор $\left(\alpha_{1}, \ldots, \alpha_{n}\right)$ и рассмотрим полученное множество как множество выполняющих векторов некоторой функции $f^{\prime}$. Функция $f^{\prime}$ зависит от $n$ переменных и имеет вес $m+1$. Если бы у функции $f^{\prime}$ существовала биюнктивная имплицента $g$, то выполнялось бы включение $E_{f^{\prime}} \subset E_{g}$; поскольку по построению $E_{f} \subset E_{f^{\prime}}$, то $E_{f} \subset E_{g}$, то есть $g$ была бы биюнктивной имплицентой функции $f$, что дает противоречие. Следовательно, у функции $f^{\prime}$ также нет биюнктивных имплицент, то есть $D_{n, m+1} \neq \varnothing$. Таким образом, если $D_{n, m} \neq \varnothing$, то $D_{n, m+1} \neq \varnothing$. Это и дает неравенство $n_{\max }(m) \leq n_{\max }(m+1)$.

Основным результатом настоящей работы является нахождение точного значения величины $n_{\max }(m)$, приведенное в $\S 3$.

\section{§ 2. Сведение к комбинаторно полным матрицам}

Отсутствие у булевой функции биюнктивных имплицент имеет наглядный комбинаторный смысл. Введем следующее определение.

Определение. Назовём $(0,1)$-матрицу $A$ размера $m \times n, n>1$, комбинаторно полной, если в любой подматрице размера $m \times 2$, образованной произвольными двумя столбцами, найдутся все 4 строки: $(0,0),(0,1),(1,0)$, $(1,1)$.

Иначе говоря, при выборе любых двух столбцов в матрице $A$ полученная подматрица должна содержать каждую из четырех возможных строк (но не обязательно только по одному разу).

Укажем несколько простых свойств комбинаторно полных матриц. Очевидно, что $m \geq 4$. Очевидно также, что инвертирование любого столбца комбинаторно полной матрицы (то есть инвертирование всех его элементов) приводит к комбинаторно полной матрице. В частности, инвертирование всей матрицы дает комбинаторно полную матрицу. Кроме того, добавление любого числа произвольных строк к комбинаторно полной матрице также дает комбинаторно полную матрицу. 
Собственно, нахождение максимального значения числа столбцов комбинаторно полной матрицы при фиксированном значении числа строк и составляет основную цель данной работы. Это обусловлено простой связью, существующей между комбинаторно полными матрицами и булевыми функциями, не имеющими биюнктивных имплицент. Матрицу, строки которой являются выполняющими векторами булевой функции $f$ от $n$ переменных, обозначим через $\tilde{E}_{f}$ :

$$
\tilde{E}_{f}=\left(\begin{array}{c}
\left(\alpha_{1}^{(1)}, \ldots, \alpha_{n}^{(1)}\right) \\
\ldots \\
\left(\alpha_{1}^{(\|f\|)}, \ldots, \alpha_{n}^{(\|f\|)}\right)
\end{array}\right),
$$

где $\left(\alpha_{1}^{(i)}, \ldots, \alpha_{n}^{(i)}\right) \in E_{f}, i=1, \ldots,\|f\|$.

Утверждение 4. Булева функиия $f$, отличная от константы, имеет биюнктивную имплиенту тогда и только тогда, когда матрица $\tilde{E}_{f}$ не является комбинаторно полной матрицей.

Доказательство. Имплицента называется элементарной, если существует ее запись в виде элементарной дизъюнкции. У функции $f$ есть биюнктивная имплицента тогда и только тогда, когда существует элементарная имплицента, зависящая от не более чем двух переменных. Действительно, если $g=\prod_{i=1}^{t}\left(x_{s_{i 1}}^{\alpha_{i 1}} \vee x_{s_{i 2}}^{\alpha_{i 2}}\right), \quad \alpha_{i j} \in\{0,1\}, i \in\{1, \ldots, t\}, j \in\{1,2\},-$ биюнктивная имплицента функции $f$, то $g^{\prime}=x_{11}^{\alpha_{11}} \vee x_{12}^{\alpha_{12}}$ является элементарной имплицентой функции $f$, поскольку $g \cdot g^{\prime}=g$ и $f \cdot g^{\prime}=(f \cdot g) \cdot g^{\prime}=f \cdot\left(g \cdot g^{\prime}\right)=f \cdot g=f$. Обратное очевидно: если есть элементарная имплицента $x_{i}^{\alpha} \vee x_{j}^{\beta}$ функции $f$, то она и является биюнктивной.

Рассмотрим элементарную дизъюнкцию, зависящую от не более чем двух переменных вида $g=x_{i}^{\alpha} \vee x_{j}^{\beta}$ ( $i$ может совпадать с $j$ : в этом случае $\alpha=\beta$ ). Эта дизъюнкция является имплицентой функции $f$ от $n$ переменных тогда и только тогда, когда для всех значений аргументов $f \cdot g=f \cdot\left(x_{i}^{\alpha} \vee x_{j}^{\beta}\right)=f$. Последнее равенство эквивалентно тому, что на всех таких векторах $\left(x_{1}, \ldots, x_{n}\right)$, что $x_{i}=\alpha \oplus 1, x_{j}=\beta \oplus 1$, должно выполняться равенство $f\left(x_{1}, \ldots, x_{n}\right)=0$; иначе говоря, среди выполняющих векторов функции $f$ нет таких, в которых на местах $i, j$ стояли бы значения $(\alpha \oplus 1, \beta \oplus 1)$ соответственно. По определению матрицы $\tilde{E}_{f}$ это происходит 
тогда и только тогда, когда в столбцах с номерами $i, j$ матрицы $\tilde{E}_{f}$ не содержится строка, равная $(\alpha \oplus 1, \beta \oplus 1)$, то есть матрица $\tilde{E}_{f}$ не является комбинаторно полной.

Доказанное утверждение обнаруживает связь между величиной $n_{\max }(m)$ и максимальным значением числа столбцов комбинаторно полной матрицы, имеющей $m$ строк. По определению $D_{n_{\max }(m), m} \neq \varnothing$, то есть существует булева функция $f$ от $n_{\max }(m)$ переменных веса $m$, не имеющая биюнктивных имплицент. Согласно утверждению 4 матрица $\tilde{E}_{f}$ является комбинаторно полной и имеет размеры $n_{\max }(m) \times m$. Следовательно, максимально возможное число столбцов в комбинаторно полной матрице, имеющей $m$ строк, не меньше $n_{\max }(m)$. Верно и обратное неравенство, однако его доказательство несколько сложнее и приведено в следующем утверждении.

Утверждение 5. Максимально возможное число столбиов в комбинаторно полной матрице, имеющей $m$ строк, равно $n_{\max }(m)$.

Доказательство. Обозначим максимально возможное число столбцов в комбинаторно полной матрице, имеющей $m$ строк, через $r(m)$. Выше показано, что $r(m) \geq n_{\max }(m)$. Докажем обратное неравенство. Пусть $A-$ комбинаторно полная матрица размера $m \times r(m)$. Если в этой матрице имеются одинаковые строки, то удалим повторы, то есть из каждой группы одинаковых строк оставим только по одной строке. Получим матрицу $A^{\prime}$ размера $m^{\prime} \times r(m), m^{\prime} \leq m$. Очевидно, что матрица $A^{\prime}$ также комбинаторно полная. В матрице $A^{\prime}$ все строки различны, поэтому эта матрица задает некоторую функцию $f$, для которой $\tilde{E}_{f}=A^{\prime}$. По утверждению 4 функция $f$ от $r(m)$ переменных веса $m^{\prime}$ не имеет биюнктивных имплицент. Следовательно, число переменных функции $f$ не превышает максимальное число переменных у функции веса $m^{\prime}$, не имеющей биюнктивных имплицент. По определению это максимальное число равно $n_{\max }\left(m^{\prime}\right)$, то есть $r(m) \leq n_{\max }\left(m^{\prime}\right)$. Далее, $n_{\max }\left(m^{\prime}\right) \leq n_{\max }(m)$ при $m^{\prime} \leq m$ по утверждению 3 , следовательно, $r(m) \leq n_{\max }(m)$.

Соединяя оба неравенства, получаем $r(m)=n_{\max }(m)$.

Доказанное утверждение позволяет нам в следующем параграфе найти значение величины $n_{\max }(m)$ именно как максимально возможного числа столбцов в комбинаторно полной матрице, имеющей $m$ строк. 


\section{§3. Значение $n_{\max }(m)$}

Всюду далее в данном параграфе рассматривается случай $m>3$.

Теорема 1. Для величины $n_{\max }(m)$ верны формульы:

- если т четно, то $n_{\max }(m)=\frac{1}{2} \cdot\left(\begin{array}{c}m \\ m / 2\end{array}\right)$,

— если т нечетно, то $n_{\max }(m)=\left(\begin{array}{c}m-1 \\ (m-3) / 2\end{array}\right)$.

Прежде чем перейти к доказательству теоремы, введем некоторые понятия и докажем вспомогательные утверждения. Напомним (см. [4]), что два булевых вектора $\alpha, \beta \in V_{m}$ называются сравнимыми, если один вектор покоординатно не меньше другого. В противном случае эти векторы называются не сравнимыми; в этом случае используем обозначение $\alpha<>\beta$. Множество векторов называется антицепью, если любые два вектора в этом множестве не сравнимы.

Определение. Векторы $\alpha, \beta \in V_{m}$ назовем сильно не сравнимыми, если не сравнимы векторы $\alpha, \beta$ и не сравнимы векторы $\bar{\alpha}, \beta$.

Очевидно, что если векторы $\alpha, \beta$ сильно не сравнимы, то множество векторов $\{\alpha, \bar{\alpha}, \beta, \bar{\beta}\}$ является антицепью.

Определение. Множество векторов $\left\{\alpha_{1}, \ldots, \alpha_{t}\right\}, \alpha_{i} \in V_{m}, i=1, \ldots, t$, образует сильную антицепь, если любые два вектора из этого множества являются сильно не сравнимыми.

Очевидно, что в сильной антицепи не могут одновременно лежать вектор и его отрицание. В терминах сильных антицепей можно сформулировать критерий комбинаторной полноты.

Утверждение 6. Матрииа А размера $m \times n$ является комбинаторно полной тогда и только тогда, когда ее столбиь образуют сильную антиuениь.

Доказательство. Пусть матрица $A$ размера $m \times n$ является комбинаторно полной. Рассмотрим пару ее столбцов $\alpha=\left(\alpha_{1}, \ldots, \alpha_{m}\right)^{T}$, $\beta=\left(\beta_{1}, \ldots, \beta_{m}\right)^{T}$. Из определения комбинаторной полноты следует, что существуют такие номера строк $i_{0}, \ldots, i_{3}$, что

$$
\left(\alpha_{i_{0}}, \beta_{i_{0}}\right)=(0,0),\left(\alpha_{i_{1}}, \beta_{i_{1}}\right)=(0,1),\left(\alpha_{i_{2}}, \beta_{i_{2}}\right)=(1,0),\left(\alpha_{i_{3}}, \beta_{i_{3}}\right)=(1,1) .
$$


Очевидно, что $\alpha<>\beta$, поскольку $\alpha_{i_{1}}<\beta_{i_{1}}$ и $\alpha_{i_{2}}>\beta_{i_{2}}$. Кроме того, поскольку $\alpha_{i_{0}} \oplus 1>\beta_{i_{0}}$ и $\alpha_{i_{4}} \oplus 1<\beta_{i_{4}}$, то $\alpha<>\bar{\beta}$. Значит векторы $\alpha, \beta$ сильно не сравнимы.

С другой стороны, предположим, что столбцы $\alpha, \beta \in V_{m}$ не являются сильно не сравнимыми. Перечислим возможные случаи:

1) $\alpha \geq \beta$, тогда в множестве $\left\{\left(\alpha_{i}, \beta_{i}\right), i=1, \ldots, m\right\}$ отсутствует пара $(0,1)$;

2) $\alpha \leq \beta$, тогда отсутствует пара $(1,0)$;

3) $\bar{\alpha} \geq \beta$, тогда отсутствует пара $(1,1)$;

4) $\bar{\alpha} \geq \beta$, тогда отсутствует пара $(0,0)$.

Во всех указанных случаях матрица $A$ не будет комбинаторно полной порядка 2.

Для максимальной мощности сильных антицепей верна оценка, легко выводимая из теоремы Шпернера (мощность любой антицепи в множестве $m$-мерных булевых векторов не превосходит $\left(\begin{array}{c}m \\ {[m / 2]}\end{array}\right)$, см., например, [4]).

Утверждение 7. Если множество векторов $\left\{\alpha_{1}, \ldots, \alpha_{t}\right\} \subset V_{m}, t \geq 2$, является сильной антицепью, то

$$
t \leq \frac{1}{2} \cdot\left(\begin{array}{c}
m \\
{[m / 2]}
\end{array}\right) .
$$

Доказательство. Пусть $\left\{\alpha_{1}, \ldots, \alpha_{t}\right\}-$ сильная антицепь. Если $t>1$, то в ней, очевидно, не содержатся векторы $(0, \ldots, 0)$ и $(1, \ldots, 1)$. Рассмотрим цепочку векторов $\alpha_{1}, \alpha_{2}, \ldots, \alpha_{t}, \bar{\alpha}_{1}, \bar{\alpha}_{2}, \ldots, \bar{\alpha}_{t}$. Так как $\|\alpha\| \notin\{0, m\}$, то $\alpha_{i}<>\bar{\alpha}_{i}, i=1, \ldots, t$. Кроме того, если $\alpha_{i}<>\alpha_{j}$, то $\alpha_{i}<>\bar{\alpha}_{j}, \bar{\alpha}_{i}<>\bar{\alpha}_{j}$, и наша последовательность образует антицепь. По теореме Шпернера ([5]) ее длина $2 t$ не превосходит $\left(\begin{array}{c}m \\ {[m / 2]}\end{array}\right]$.

Чтобы теперь доказать теорему 1 , надо показать, что для четного $m$ оценка из утверждения 7 достижима; для нечетного значения $m$ необходимо уточнить оценку и также доказать ее достижимость.

Для уточнения оценки в случае нечетного $m$ воспользуемся теоремой Эрдёша-Ко-Радо (см., например, [6]). В одной из эквивалентных форм ее 
формулировка выглядит следующим образом. Пусть семейство подмножеств $A_{1}, \ldots, A_{t}$ конечного множества $X$ мощности $m$ обладает следующими тремя свойствами: существует такое $k, k \leq\left[\frac{m}{2}\right]$, что $\left|A_{i}\right| \leq k$ для любого $i=1, \ldots, t$; для любых $i$ и $j, i \neq j, A_{i} \cap A_{j} \neq \varnothing$; для любых $i$ и $j, i \neq j$, $A_{i} \not \subset A_{j}$. Тогда верно неравенство $t \leq\left(\begin{array}{c}m-1 \\ k-1\end{array}\right)$. Чтобы применить этот результат, возьмем натуральное число $m \geq 4$ нечетным и рассмотрим произвольную сильную антицепь $\left\{\alpha_{1}, \ldots, \alpha_{t}\right\}, \alpha_{i} \in V_{m}, i=1, \ldots, t$. Как указывалось выше, если два вектора сильно не сравнимы, то инвертирование одного вектора (или обоих) приводит также к сильно не сравнимой паре, поэтому, инвертируя векторы в сильной антицепи $\left\{\alpha_{1}, \ldots, \alpha_{t}\right\}$, добиваемся того, чтобы вес каждого вектора $\alpha_{i}, i=1, \ldots, t$, не превышал $(m-1) / 2$. Представим каждый из двоичных векторов $\alpha_{i} \in\left\{\alpha_{1}, \ldots, \alpha_{t}\right\}$ (размерности $m$ ) как характеристический вектор подмножества $I\left(\alpha_{i}\right)$ в конечном множестве $X=\left\{x_{1}, \ldots, x_{m}\right\}: x_{s} \in I\left(\alpha_{i}\right)$ тогда и только тогда, когда $s$-я координата вектора $\alpha_{i}$ равна 1. Так как $\left\|\alpha_{i}\right\| \leq(m-1) / 2$, то $\left|I\left(\alpha_{i}\right)\right| \leq(m-1) / 2$ для всех $i=1, \ldots, t$. Пусть $\alpha_{i}, \alpha_{j}$ - два различных вектора из семейства $\left\{\alpha_{1}, \ldots, \alpha_{t}\right\}$. По утверждению 6 в матрице $T$, составленной из двух столбцов $\left(\alpha_{i}, \alpha_{j}\right)$, есть все возможные строки: $(0,0),(1,0),(0,1)$ и $(1,1)$. Наличие строк $(1,0),(0,1)$ означает, что ни одно из множеств $I\left(\alpha_{i}\right), I\left(\alpha_{j}\right)$ не лежит в другом. Наличие строки $(1,1)$ означает, что $I\left(\alpha_{i}\right) \cap I\left(\alpha_{j}\right) \neq \varnothing$. Таким образом, набор множеств $I\left(\alpha_{1}\right), \ldots, I\left(\alpha_{t}\right)$ удовлетворяет условиям используемой теоремы при $k=(m-1) / 2$. Следовательно, доказано следующее.

Утверждение 8. Если т нечетно и множество векторов $\left\{\alpha_{1}, \ldots, \alpha_{t}\right\}$, $\alpha_{i} \in V_{m}, i=1, \ldots, t$, является сильной антицепью, то

$$
t \leq\left(\begin{array}{c}
m-1 \\
(m-3) / 2
\end{array}\right) .
$$

Докажем еще несколько простых лемм. 
Лемма 2. Если т четно и различные векторы $\alpha$ и $\beta$ таковы, что $\|\alpha\|=\|\beta\|=m / 2$, то либо $\alpha=\bar{\beta}$, либо $\alpha$ и $\beta$ сильно не сравнимы.

Доказательство. Обозначим через $A$ множество номеров координат, которые в векторе $\alpha$ равны единице; через $B$ - аналогичное множество для вектора $\beta$. По условию $|A|=|B|=m / 2$ и $A \neq B$. Если $\alpha \neq \bar{\beta}$, то $A \cap B \neq \varnothing$. Следовательно, существуют $i_{0} \in A \cap B, i_{1} \in A \backslash B, i_{2} \in B \backslash A$ и $i_{3} \in(1, \ldots, m) \backslash(A \cup B)$. Координата с номером $i_{1}$ в векторе $\alpha$ равна 1 , а в векторе $\beta$ равна 0 . Координаты с номером $i_{2}$ соответственно равны 0 и 1 . Поэтому векторы $\alpha$ и $\beta$ не сравнимы. Координаты с номером $i_{0}$ равны 1 ; координаты с номером $i_{3}$ равны 0 . Поэтому векторы $\bar{\alpha}$ и $\beta$ не сравнимы, то есть $\alpha$ и $\beta$ сильно не сравнимы.

Для векторов $\alpha$ и $\beta$ из $V_{m}$ через $\alpha \cdot \beta$ обозначим булев вектор размерности $m$, полученный перемножением координат векторов $\alpha$ и $\beta$.

Лемма 3. Если т нечетно и два различных вектора $\alpha$ и $\beta$ таковы, что $\|\alpha\|=\|\beta\|=\frac{m-1}{2}$, то $\alpha$ и $\beta$ сильно не сравнимы тогда и только тогда, когда $\alpha \cdot \beta \neq(0, \ldots, 0)$.

Доказательство. Обозначим $\alpha=\left(\alpha_{1}, \ldots, \alpha_{m}\right)$ и $\beta=\left(\beta_{1}, \ldots, \beta_{m}\right)$. Так как веса векторов $\alpha$ и $\beta$ одинаковы, то очевидно, что $\alpha<\beta$. Пусть $\alpha \cdot \beta=(0, \ldots, 0)$, то есть нет координаты, которая одновременно и в $\alpha$, и в $\beta$ равна 1. Поскольку $\|\alpha\|=\|\beta\|=\frac{m-1}{2}$, то существует единственная такая координата $i$, что $\left(\alpha_{i}, \beta_{i}\right)=(0,0)$ и $\left(\alpha_{j}, \beta_{j}\right) \in\{(0,1),(1,0)\}$ для всех $j \in\{1, \ldots, m\} \backslash\{i\}$. Следовательно, $\left(\alpha_{i}, \bar{\beta}_{i}\right)=(0,1)$ и $\left(\alpha_{j}, \bar{\beta}_{j}\right) \in\{(0,0),(1,1)\}$ для всех $j \neq i$. Тогда $\alpha<\bar{\beta}$, то есть свойство сильной несравнимости отсутствует.

Пусть теперь $\alpha \cdot \beta \neq(0, \ldots, 0)$, то есть существует такая координата $v$, что $\left(\alpha_{v}, \beta_{v}\right)=(1,1)$. Поскольку $\|\alpha\|=\|\beta\|=\frac{m-1}{2}$, найдется такая координата $l$, что $\left(\alpha_{l}, \beta_{l}\right)=(0,0)$. Значит, $\left(\bar{\alpha}_{v}, \beta_{v}\right)=(0,1)$, а $\left(\bar{\alpha}_{l}, \beta_{l}\right)=(1,0)$. Это означает, что $\bar{\alpha}<>\beta$, то есть векторы $\alpha$ и $\beta$ сильно не сравнимы.

Перейдем к доказательству теоремы 1. 
Доказательство теоремы 1. Пусть $m$ четно. Возьмем все векторы размерности $m$ и веса $m / 2$. Введем на этом множестве отношение эквивалентности: векторы $\alpha$ и $\beta$ эквивалентны, если $\alpha=\bar{\beta}$. Очевидно, что каждый класс эквивалентности имеет мощность 2. Взяв по одному представителю из каждого класса, получим некоторое множество $R$. По лемме 2 это множество является сильной антицепью. Кроме того, мощность множества $R$ равна $\frac{1}{2} \cdot\left(\begin{array}{c}m \\ m / 2\end{array}\right)$, то есть верхняя оценка из утверждения 7 в случае четного $m$ достижима.

Пусть теперь $m$ нечетно. Рассмотрим множество $P$ векторов размерности $m$ и веса $\frac{m-1}{2}$, у которых первая координата равна 1 . По лемме 3 множество $P$ будет сильной антицепью; мощность $P$ равна $\left(\begin{array}{c}m-1 \\ (m-3) / 2\end{array}\right)$. Значит, верхняя оценка для мощности сильной антицепи из утверждения 8 также достижима, что завершает доказательство теоремы.

\section{§ 4. Следствия из теоремы 1}

Из теоремы 1 можно вывести несколько простых следствий. Поскольку функция $n_{\max }(m)$ описывается разными формулами для четных и нечетных значений $m$, то для простоты рассуждений отметим один элементарный факт. Пусть $\left\{a_{s}, s \in \mathbb{N}\right\}-$ произвольная последовательность вещественных чисел. Очевидно что, если $\lim _{s \rightarrow \infty} a_{2 s}=t$ и $\lim _{s \rightarrow \infty} a_{2 s+1}=t$, где $t \in \mathbb{R}$, то $\lim _{s \rightarrow \infty} a_{s}=t$.

Сначала отметим, что увеличение числа строк в комбинаторно полной матрице на 1 отражается примерно двукратным увеличением максимально возможного числа столбцов. Более строго сформулируем это в следующем утверждении.

Следствие 2. Для любого $m \geq 4$

$$
\frac{n_{\max }(m+1)}{n_{\max }(m)} \underset{m \rightarrow \infty}{\longrightarrow} 2
$$


Доказательство. При $s \geq 2$

$$
\frac{n_{\max }(2 s+1)}{n_{\max }(2 s)}=\frac{\left(\begin{array}{c}
2 s \\
s-1
\end{array}\right)}{\frac{1}{2}\left(\begin{array}{c}
2 s \\
s
\end{array}\right)}=2 \cdot \frac{s}{s+1} \underset{s \rightarrow \infty}{\longrightarrow} 2,
$$

и при $s \geq 3$

$$
\frac{n_{\max }(2 s)}{n_{\max }(2 s-1)}=\frac{\frac{1}{2}\left(\begin{array}{c}
2 s \\
s
\end{array}\right)}{\left(\begin{array}{c}
2 s-2 \\
s-2
\end{array}\right)}=\frac{2 s-1}{s-1}=2+\frac{1}{s-1} \underset{s \rightarrow \infty}{\longrightarrow} 2
$$

Следовательно, $\frac{n_{\max }(m+1)}{n_{\max }(m)} \underset{m \rightarrow \infty}{\longrightarrow} 2$.

Следствие 3. При $m \geq 4$ функциия $n_{\max }(m)-$ строго возрастающุая.

Доказательство. Для любого $s \quad$ верно $\frac{n_{\max }(2 s+1)}{n_{\max }(2 s)}=2 \cdot \frac{s}{s+1}>1$ и $\frac{n_{\max }(2 s)}{n_{\max }(2 s-1)}=2+\frac{1}{s-1}>1$.

Теорема 1 показывает, что при четных и нечетных значениях $m$ функция $n_{\max }(m)$ обладает одинаковой асимптотикой.

Следствие 4. Если $m \rightarrow \infty$, то $n_{\max }(m) \sim \frac{2^{m}}{\sqrt{2 \pi m}}$.

Доказательство. Воспользуемся формулой Стирлинга и найдем асимптотику функции $\left(\begin{array}{c}2 s \\ s\end{array}\right)$. Имеем при $s \rightarrow \infty$

$$
\left(\begin{array}{c}
2 s \\
s
\end{array}\right)=\frac{(2 s) !}{(s !)^{2}} \sim \frac{\left(\frac{2 s}{e}\right)^{2 s} \cdot \sqrt{4 \pi s}}{\left(\left(\frac{s}{e}\right)^{s} \cdot \sqrt{2 \pi s}\right)^{2}}=\frac{2^{2 s}}{\sqrt{\pi s}}
$$




$$
\begin{gathered}
\text { Отсюда получаем, что } n_{\max }(2 m)=\frac{1}{2} \cdot\left(\begin{array}{c}
2 m \\
m
\end{array}\right) \sim \frac{2^{2 m}}{\sqrt{2 \pi(2 m)}} \text { при } m \rightarrow \infty \text { и } \\
n_{\max }(2 m+1)=\left(\begin{array}{c}
2 m \\
m-1
\end{array}\right)=\frac{(2 m) !}{m ! \cdot m !} \cdot \frac{m}{m+1} \sim \frac{(2 m) !}{m ! \cdot m !}=\left(\begin{array}{c}
2 m \\
m
\end{array}\right) \sim \frac{2^{2 m}}{\sqrt{\pi m}} \sim \frac{2^{2 m+1}}{\sqrt{2 \pi(2 m+1)}} .
\end{gathered}
$$

Следовательно, $n_{\max }(m) \sim \frac{2^{m}}{\sqrt{2 \pi m}}$ при $m \rightarrow \infty$.

В заключение параграфа рассмотрим вопрос о количестве сильных антицепей длины $n_{\max }(m)$.

Утверждение 9. Число сильных антицепей из $n_{\max }(m)$ векторов размерности т равно $2^{n_{\max }(m)}$, если $m$ четно, и $m \cdot 2^{n_{\max }(m)}$, если $m$ нечетно.

Доказательство. Для доказательства воспользуемся известными фактами об антицепях, на которых достигаются оценки в теореме Шпернера и Эрдёша-Ко-Радо (см., например, [3]). Пусть $\alpha_{1}, \ldots, \alpha_{t}$ - сильная антицепь из булевых векторов размерности $m$ максимальной длины $t=n_{\max }(m)$.

Пусть $m$ четно, тогда $n_{\max }(m)=\frac{1}{2}\left(\begin{array}{c}m \\ m / 2\end{array}\right)$ по теореме 1. Рассмотрим множество векторов $\left\{\beta_{1}, \ldots, \beta_{2 t}\right\}=\left\{\alpha_{1}, \ldots, \alpha_{t}, \bar{\alpha}_{1}, \ldots, \bar{\alpha}_{t}\right\}$. Из определения сильной несравнимости очевидно следует, что $\left\{\beta_{1}, \ldots, \beta_{2 t}\right\}$ - антицепь длины $2 t=\left(\begin{array}{c}m \\ m / 2\end{array}\right)$. Известно ([5]), что при четном $m$ существует единственная антицепь максимальной длины $\left(\begin{array}{c}m \\ m / 2\end{array}\right)$ : это антицепь, составленная из всех векторов веса $\frac{m}{2}$. Следовательно, набор $\left\{\beta_{1}, \ldots, \beta_{2 t}\right\}=\left\{\alpha_{1}, \ldots, \alpha_{t}, \bar{\alpha}_{1}, \ldots, \bar{\alpha}_{t}\right\}$ и является такой антицепью. Таким образом, получаем, что любая сильная антицепь максимальной длины получается путем выбора среди всех векторов веса $\frac{m}{2}$ половины так, чтобы вектор и его отрицание одновременно не выбирались. 
Как и в доказательстве теоремы 1 , все множество векторов длины $\frac{m}{2}$ разобьем на $\frac{1}{2}\left(\begin{array}{c}m \\ m / 2\end{array}\right)$ непересекающихся классов по два элемента в каждом - каждый класс содержит некоторый вектор и его отрицание. Выбор сильной антицепи мощности $\frac{1}{2}\left(\begin{array}{c}m \\ m / 2\end{array}\right)$ векторов эквивалентен выбору представителя от каждого класса. Следовательно, всего существует $2^{\frac{1}{2}\left(\begin{array}{c}m \\ m\end{array}\right)}$ сильных антицепей указанной мощности.

Пусть теперь $m$ нечетно и $t=n_{\max }(m)=\left(\begin{array}{c}m-1 \\ (m-3)\end{array}\right)$ по теореме 1. Из определения очевидно, что инвертирование любых векторов в $\alpha_{1}, \ldots, \alpha_{t}$ (инвертирование вектора есть инвертирование каждой его координаты) приводит также к сильной антицепи. Поэтому инвертированием векторов в $\alpha_{1}, \ldots, \alpha_{t}$ добьемся того, что их веса не превосходили $\frac{(m-1)}{2}$. Как известно ([6]), при нечетном $m$, если длина антицепи совпадает с оценкой из теоремы Эрдёша-Ко-Радо, то все векторы антицепи имеют одинаковый вес, равный $k$ (в нашем случае мы применяем эту теорему при $\left.k=\frac{(m-1)}{2}\right)$, и найдется такая координата, что во всех векторах из этой антицепи данная координата равна 1. Поскольку сильная антицепь, в частности, является антицепью, это означает, что $\alpha_{1}, \ldots, \alpha_{t}$ имеет вид (*): одна из координат (назовем ее $i_{0}$ ) в каждом из векторов $\alpha_{j}, j=1, \ldots, t$, равна 1 ; векторы $\alpha_{1}, \ldots, \alpha_{t}$ получаются распределением по $m-1$ оставшимся координатам $\frac{m-3}{2}$ единиц всеми возможными способами.

Таким образом, всякая сильная антицепь максимальной длины получается из сильной антицепи вида (*) инвертированием части векторов. Сильных антицепей вида (*) ровно $m$ (координату $i_{0}$ можно выбрать $m$ способами). Инвертировать можно любое подмножество из $t=\left(\begin{array}{c}m-1 \\ (m-3)\end{array}\right)$ век- 
торов, то есть $2^{t}$ способов. Следовательно, всего существует $m \cdot 2^{\left(\begin{array}{c}m-1 \\ (m-3)\end{array} / 2\right)}$ сильных антицепей максимальной длины.

Пользуясь утверждениями 3 и 6 и формулами для числа сильных антицепей максимальной длины, можно вычислить количества булевых функций $f\left(x_{1}, \ldots, x_{n}\right)$, удовлетворяющих какому-нибудь из условий:

1) $n=n_{\max }(m)$ при некотором натуральном $m$;

2) никакая биюнктивная функция не является имплицентой функции $f$;

3 ) функция $f$ имеет минимально возможный вес (равный $m$ ) среди всех булевых функций, удовлетворяющих условиям 1 и 2.

Отметим, что число булевых функций от $n=n_{\max }(m)$ переменных и веса $m$ равно $\left(\begin{array}{c}2^{n_{\max }(m)} \\ m\end{array}\right) \approx \frac{2^{m \cdot n_{\max }(m)}}{m !}$ (асимптотическая формула верна, поскольку $\left.2^{n_{\max }(m)}>>m\right)$.

Утверждение 10. Число булевых функиий, удовлетворяющих условиям $1-3$, равно $\frac{2^{n_{\max }(m)} \cdot n_{\max }(m) !}{m !}$, если $m$ четно, и $\frac{2^{n_{\max }(m)} \cdot m \cdot n_{\max }(m) !}{m !}$, если $m$ нечетно.

Доказательство. Каждая сильная антицепь из $n_{\max }(m)$ векторов дает $n_{\max }(m)$ ! различных комбинаторно полных матриц за счет перестановки векторов (столбцов матрицы). Очевидно также, что две различные сильные антицепи не могут образовать одну и ту же комбинаторно полную матрицу. Поэтому общее число комбинаторно полных матриц размера $m \times n_{\max }(m)$ порядка 2 равно $2^{n_{\max }(m)} \cdot n_{\max }(m)$ ! для четного $m$ и $2^{n_{\max }(m)} \cdot m \cdot n_{\max }(m)$ ! для нечетного $m$.

Остается заметить, что из полученного в доказательстве утверждения 9 описания вида сильных антицепей максимальной длины и для четных и для нечетных значений $m$ в любой комбинаторно полной матрице размера $m \times n_{\max }(m)$ нет одинаковых строк. А любая перестановка строк в матрице $\tilde{E}_{f}$ приводит к той же булевой функции $f$. Следовательно, каждой булевой функции, удовлетворяющей условиям $1-3$, соответствует ровно $m$ ! комбинаторно полных матриц размера $m \times n_{\max }(m)$ порядка 2. Отсюда получаем искомое значение числа таких функций. 


\section{Список литературы}

1. Горшков С. П. О сложности распознавания мультиаффинности, биюнктивности, слабой положительности и слабой отрицательности булевых функций // Обозрение прикладной и промышленной математики. 1997. - T. 4. Вып. 2. - С. 216-237.

2. Schaefer T. Complexity of satisfiability problems // Proceedings of the 10 Annual ACM Symposium on Theory of Computing. - 1978. - P. 216-226.

3. Горшков С. П. Применение теории NP-полных задач для оценки сложности решения систем булевых уравнений // Обозрение прикладной и промышленной математики. - 1995. - Т. 2. Вып. 3. - С. 325-398.

4. Яблонский С. В. Введение в дискретную математику. - М.: Наука, 1986.

5. Сачков В. Н. Введение в комбинаторные методы дискретной математики. - М.: Наука, 1982.

6. Райгородский $A$. М. Системы общих представителей и их приложения в геометрии. - М.: МЦНМО, 2006. 